\title{
Verfassungsrechtliche Aspekte der Krise in Venezuela
}

\author{
Von Nicole Monleón, Hamburg*
}

\section{Einführung}

Venezuela durchlebte 2002/2003 eine schwere politische Krise und erwies sich in dieser Zeit als nicht regierbar. Wiederholte Streikaufrufe, Proteste und Gegenproteste, die in ihrer Mehrzahl friedlich verliefen sowie Militärangehörige im zivilen Ungehorsam prägten das Tagesgeschehen. Die Opposition forderte den sofortigen Rücktritt des Präsidenten Hugo Rafael Chávez Frías und suchte dieser Forderung durch einen landesweiten Generalstreik zwischen Dezember 2002 und Februar 2003 Nachdruck zu verleihen. Bestreikt und zeitweilig lahm gelegt wurde insbesondere das staatliche Erdölunternehmen Petróleos de Venezuela S.A. (PDVSA). Trotz Versorgungsengpässen in der Bevölkerung und verheerenden wirtschaftlichen Folgen trat Hugo Chávez nicht von seinem Amt zurück. Chávez gab sich nach Beendigung des Generalstreiks, den er als Kapitulation der Opposition wertete, als strahlender Sieger und betont zuversichtlich. Seitdem verfolgt das 2001 ins Leben gerufene Oppositionsbündnis Coordinadora Democrática das Ziel der Absetzung Chávez mit anderen Mitteln, aber nicht weniger entschlossen.

Der vorliegende Beitrag untersucht, warum die Opposition in der einstigen lateinamerikanischen Musterdemokratie einen demokratisch gewählten Präsidenten mit aller Macht zu Fall bringen möchte. Er widmet sich den Ursachen der Krise aus der Zeit vor dem Regierungsantritt des amtierenden Präsidenten Chávez, um die heutige politische Landschaft Venezuelas verstehen $\mathrm{zu}$ helfen. Anschließend geht es vor dem einschlägigen verfassungsrechtlichen Hintergrund um die Gründe für die Eskalation zwischen Regierung und Opposition. Den Abschluss bilden die diskutierten Lösungen zur demokratischen Beilegung der Krise.

* Dieser Aufsatz beruht auf einem Vortrag im Konzil des Max-Planck-Instituts für ausländisches und internationales Privatrecht, den die Verfasserin gemeinsam mit Rosario Then de Lammerskötter am 24. Februar 2003 gehalten hat.

Abgekürzt zitiert werden: Allan Randolph Brewer-Carías, La Constitución de 1999, 3. A., Caracas 2001 (zitiert: Brewer-Carías); Laura Neuman und Jennifer McCoy: Observando el cambio político en Venezuela. La Constitución Bolivariana y las elecciones 2000, Informe final del Centro Carter, febrero 2001, <http://www.cartercenter.org/documents/696.pdf> (zitiert: Neuman/McCoy). Nicht anders gekennzeichnete Übersetzungen stammen von der Verfasserin. 


\section{A. Vorgeschichte}

Die tieferen Ursachen der Krise Venezuelas liegen einige Jahre zurück ${ }^{1}$. Nach der Militärdiktatur von General Marcos Pérez Jiménez (1953-1958) konsolidierte sich in Venezuela eine Parteiendemokratie. Die drei wichtigsten Parteien AD, COPEI und URD ${ }^{2}$ schlossen 1958 den Pakt des Punto Fijo . Darin bekannten sie sich ausdrücklich zur Demokratie, einigten sich auf die Grundlagen der künftigen Politik und die Anerkennung des nächsten Wahlergebnisses ${ }^{3}$. Mittelbares Ergebnis des Paktes war 1961 eine neue Verfassung, auf deren Grundlage sich die Demokratie in Venezuela festigte ${ }^{4}$. Die kommenden Jahre etablierten ein christlich-sozialdemokratisches Zweiparteiensystem, in dessen Rahmen AD und COPEI sich in der Regierung abwechselten.

Die Ölkrise von 1973 bescherte Venezuela explosionsartig ansteigende Erdöleinnahmen, die von der Regierung in populistischer Manier ausgeschüttet wurden. Die Verstaatlichung der Stahl- und Erd ölindustrie 1975 und 1976 verstärkte den populistischen Trend. Den beiden großen Parteien gelang es trotz immenser Erdöleinnahmen nicht, die zur Diversifizierung der Wirtschaft erforderlichen Reformen durchzuführen. Das brachte die nationale Wirtschaft in die unmittelbare Abhängigkeit von der Ölpreisentwicklung. Sinkende Erdölpreise auf dem Weltmarkt, eine Fördermengenreduzierung der Organi sation Erdöl exportierender Länder (OPEC), die stagnierende Landwirtschaft und eine hohe Importabhängigkeit verursachten eine seit Anfang der 80er Jahre anhaltende Wirtschafts krise. Die Auslandsverschuldung stieg unkontrolliert an, und die Einfüh rung der Devisen- und Preiskontrolle, gepaart mit der notwendigen und zugleich drasti schen Abwertung des Bolivar am 18. Februar 1983 (viernes negro), beschleunigten den freien Fall der venezolanischen Wirtschaft. Inflation, Arbeitslosig keit und Massenarmut stürzten das Land zusätzlich in eine soziale Krise.

Bedingt durch Inkompetenz, Ineffizienz und Korruption der Staatsgewalten verlor das politische System auf der Grundlage der Verfassung von 1961 an Glaubwürdigkeit und damit auch den Rückhalt in der Bevölkerung. An dem Staatsstreichversuch vom Februar

Eingehend hierzu: José Vicente Carrasquero Aumaitre, Venezuela: Demokratie in der Krise, Rio de Janeiro: Konrad-Adenauer-Stiftung 2002, 7-20 (Europa América Latina: Analysen und Berichte, Nr. 9).

2 Die sozialdemokratische Acción Democrática (AD), das christlich-demokratische Comité de Organisación Política Electoral del Pueblo Independiente (COPEI) und die liberale Unión Republicana Democrática (URD).

Siehe zum Pacto de Punto Fijo: <http://www.analitica.com/bitblioteca/venezuela/punto_fijo. asp>.

4

Die Verfassung wurde veröffentlicht in der Gaceta Oficial Nr. 662 Extraordinario vom 23. Januar 1961. Zur Entwicklung des demokratischen Staates Venezuela: Norbert Lösing, Verfassungsentwicklung in Venezuela: JöR 37 (1988) 551, $561 \mathrm{ff.}$ 
1992 gegen die Regierung von Carlos Andrés Pérez war auch Oberstleutnant Chávez maßgebend beteiligt. Die Revolte ließ Chavez zum Helden derjenigen avan cieren, die sich gegen die korrupte politische Elite des Landes auflehnten. Wenige Tage nach dem gescheiterten Militärputsch wurde der Gedanke einer demokratischen Rekonstruktion des politischen Systems durch die Einberufung einer Verfassunggebenden Versammlung öffentlich gemacht ${ }^{5}$. Im November desselben Jahres folgte ein zivil-militäri scher Putschver such hochrangiger Offiziere, regierungsgegnerischer Gruppierungen und Mitglieder revo lutionärer Organisationen. Ein Jahr später wurde Carlos Andrés Pérez wegen Veruntreuung und Unterschlagung des Amtes enthoben ${ }^{6}$. Im Jahr 1994 begnadigte der neu gewählte Präsident, Rafael Caldera, die Verantwortlichen des Militärputsches von 1992, darunter Hugo Chávez, der zu diesem Zeitpunkt zwei Jahre seiner Haftstrafe verbüßt hatte. Caldera gelang es zwar; Venezuela politisch zu stabilisieren; die wirtschaftliche Rezession setzte sich indessen fort und vertiefte sich 1994 während einer schweren Finanz- und Bankenkrise. Erst 1996/1997 begann das unter Caldera einge leitete Stabilisierungs- und Strukturanpassungsprogramm des IWF (Agenda Venezuela) die wirtschaftliche Lage des Landes zu verbessern.

\section{B. Gründe der innenpolitischen Eskalation}

Die Wende sollte der Hoffnungsträger Hugo Chávez bringen. Im Jahre 1998 wurde er als Kandidat des linksnationalistischen Wahlbündnisses Polo Patriótico unter Einschluss des Movimiento Quinta República (MVR) eindrucksvoll mit einer Mehrheit von 56\% zum Präsidenten gewählt ${ }^{7}$. Damit legitimierten die Venezolaner seine populistisch geprägte Politik und die von ihm als ,friedliche bolivarianische Revolution“ bezeichnete umfassende Erneuerung des Landes. Bei seiner Vereidigung am 2. Februar 1999 kündigte Chávez an, eine neue Republik zu gründen, die „Bolivarianische Republik“, und dieser eine Verfassung im Einklang mit der neuen Zeit zu geben ${ }^{8}$. Dem Regierungsprogramm zufolge waren integrale Bestandteile der neuen Republik die Einführung der bolivarianischen Demokratie, die Steigerung der wirtschaftlichen Produktivität, die Herstellung sozialer Gerechtigkeit,

Siehe dazu: Brewer-Carías, S. 21 und Lösing, op. cit. (Fn. 4) 551f.

Die Verfassung von 1961 enthielt in Art. 150 Nr. 8 i.V.m. Art. 215 Nr. 1 noch das Recht des Senates, mit der Stimmenmehrheit seiner Mitglieder und nach vorherigem Beschluss des Obersten Gerichts über das Vorliegen hinreichender Gründe die Präsidentenanklage zu genehmigen und ihn damit zu suspendieren (Impeachment-Verfahren).

Die Wahlbeteiligung betrug 64\%. Eingehend: Friedrich Welsch / Nikolaus Werz, Der Wahlsieg und der Regierungsbeginn von Hugo Chávez Frías in Venezuela: Universität Rostock, Rostock 1999, S. 35ff. Siehe auch: Michael Lingenthal, Venezuela nach den Wahlen. Vor einer ungewissen Zukunft: KAS-AI, Nr. 3/99, 63, 68; Peter Rösler, Lateinamerika Jb. (1999) 199, 208; $<$ http://www.georgetown.edu/pdba/Elecdata/Venezuela/pre98.html>.

Siehe: <http://www.analitica.com/bitblioteca/hchavez/juramento.asp >. 
die Erschließung und Konsolidierung des Staatsgebietes sowie die Stärkung der Souveränität und multipolaren Integration ${ }^{9}$. Die Vertiefung und Eskalation der Krise sind aufs engste mit dem Prozess der Umsetzung dieser Ziele verbunden.

\section{Errichtung der bolivarianischen Demokratie}

Politische Zielsetzung der Regierungskoalition war die Errichtung der bolivarianischen Demokratie in Venezuela ${ }^{10}$. Hierzu bedurfte es gemäß dem Regierungsprogramm einer friedlichen demokratischen Revolution, einer neuen Verfassung, der Etablierung eines Übergangsregimes und der Institutionalisierung der neuen Republik ${ }^{11}$.

\section{Demokratische Revolution und neue Verfassung}

Die Zielsetzung der demokratischen Revolution bestand darin, durch demokratische Mittel die bestehenden politischen Institutionen aufzuheben und umzugestalten. Der bestehende institutionelle Rahmen war von einer Elite der traditionellen Parteien beherrscht worden. Diese politische Machtkonzentration, gepaart mit der staatsorganisationsrechtlichen Befugnis über die Verwendung der Erdöleinkünfte zu entscheiden, hatten Korruption, Vetternwirtschaft und Klientelismus gefördert und zugleich zur Erosion der demokratischen Entwicklung geführt. Als erster Schritt gegen die mit den Institutionen fest verwobene herrschende Parteielite wurde eine Verfassunggebende Nationalversammlung (Asamblea Nacional Constituyente - ANC) einberufen, welche mit einer neuen Verfassung die Staatsgewalten relegitimieren sollte.

\section{a) Einberufung einer Verfassunggebenden Nationalversammlung}

Die damals geltende Verfassung ${ }^{12}$ aus dem Jahre 1961 enthielt in Art. 245 ein Verfahren zur Änderung (enmienda) und in Art. 246 eines zur Totalrevision (reforma) der Verfassung. Die Einberufung einer Verfassunggebenden Nationalversammlung (ANC) war

Das Regierungsprogramm von Chávez ist abrufbar unter: <http://www.mpd.gov.ve/prog-gob/ prog_gob2/indice.htm>.

Regierungsparteien waren: MVR, MÁS (Movimiento al Socialismo), PPT (Patria Para Todos) und PC (Partido Comunista). Siehe zur Bilanz der Regierungsarbeit auch: Carrasquero Aumaitre, S. 32-37.

11 Siehe hierzu: <http://www.mpd.gov.ve/prog-gob/prog_gob2/e_politico.htm>.

12 Constitución Nacional von 1961 (CN), veröffentlicht in der Gaceta Oficial Nr. 662 Extraordinario vom 23. Januar 1961. 
demgegenüber nicht vorgesehen. Bereits im Wahlkampf war eine kontroverse Diskussion über die Einberufung einer ANC entbrannt. Nach Ansicht von Chávez war ein Konsultationsreferendum, das neben dem „Ob“ der Einberufung auch die Verfahrensweise zur Nominierung und Wahl der Delegierten sowie die Aufgabe und den Handlungsspiel raum der ANC umfassen würde, ausreichende Grundlage für deren Einberufung. Chávez' Hauptargument: Die Volkssouveränität nach Art. 4 CN (1961) ${ }^{13}$. Nach der gegenteiligen Auffassung hätte die Einberufung einer ANC ebenso wie deren Handlungsspielraum verfassungsrechtlich geregelt sein müssen. Die notwendige Verfassungsreform hätte zuvor dem Parlament im Wege des Kon sultationsreferendums aufgegeben werden können. Hauptargument: Die Unverletzbarkeit der Verfassung, Art. 250 CN (1961) ${ }^{14}$.

Zur Klärung dieser Rechtsfrage wurden noch im Jahre 1998 zwei Verfahren zur Auslegung von Gesetzen (recurso de interpretación) eingeleitet ${ }^{15}$. Vorgelegt wurde Art. 181 Ley Orgánica de Sufragio y sobre la Participación Política (LOSPP) ${ }^{16}$ und Art. 4 CN (1961) i.V.m. Art. 181 LOSPP. Der Oberste Gerichtshof (CSJ) ${ }^{17}$ entschied am 19. Januar 1999 in zwei Urteilen positiv über die Durchführung eines Konsultationsreferendums zur Einberufung einer Verfassunggebenden Nationalversammlung auf der Grundlage des Art. 181 LOSPP $^{18}$. Die CSJ unterschied im ersten Urteil deutlich zwischen dem in Art. 181 LOSPP geregelten Referendum und einer plebiszitären Befragung ${ }^{19}$. Im Hinblick auf die Wirkung

Art. 4 CN (1961): „Die Souveränität ruht im Volke, welches sie durch Wahlen und über Organe der Staatsgewalt ausübt“". Deutsche Übersetzung von Arnold Spitta und Albrecht Weber, hrsg. von Veba Öl, Gelsenkirchen 1998.

Art. 250 CN (1961): „Die Verfassung verliert ihre Wirkung nicht, wenn sie durch einen Gewaltakt nicht mehr befolgt oder auf irgendeinem anderen Wege als dem, der von ihr vorgesehen ist, außer Kraft gesetzt wird“. Deutsche Übersetzung, op.cit. (Fn. 13).

Gemäß Art. 42 Nr. 24 der Ley Orgánica de la Corte Suprema de Justicia (LOCSJ) besteht die Möglichkeit dem Obersten Gerichtshof Gesetzestexte zur Auslegung vorzulegen. Die LOCSJ wurde veröffentlicht in der Gaceta Oficial Nr. 1.893 Extraordinario vom 30. Juli 1976.

Art. 181 LOSPP: „... die Durchführung eines Referendums, mit dem Ziel der Befragung der Wähler über Entscheidungen von besonderer nationaler Tragweite“.

Oberster Gerichtshof und zugleich auch Verfassungsgerichtshof war damals noch die Corte Suprema de Justicia (CSJ). Seit dem In-Kraft-Treten der neuen Verfassung von 1999 ist es der Tribunal Supremo de Justicia (TSJ).

Die Urteile Nr. 17 und Nr. 18 der CSJ/Sala Político-Administrativa (SPA) sind abgedruckt bei: Jur.C.S.J. (Pierre Tapia) 1999, Nr. 1, 153-167 und Nr. 2 168-179 respektive. Sie finden sich auch unter: <http://www.analitica.com/bitblioteca/csj/fallo17.asp > und <http://www.analitica.com /bitblioteca/csj/fallo18.asp>. Kritisch zu den Urteilen der CSJ: Alessandro Pace, La Muerte de una Constitución: Revista Española de Derecho Constitucional 1999, SEP-DIC, 19 (57) 271-283.

Urteil Nr. 17 der CSJ/Sala Político-Administrativa vom 19. Januar 1999. Dieses Urteil wurde mit dem Urteil vom 18. März 1999 bestätigt, in dem die CSJ den eminent konsultativen Charakter des Referendums ausdrücklich und in Abgrenzung zu Befragungen mit autorisierendem Charakter feststellte. Das Urteil Nr. 271 der CSJ/Sala Político-Administrativa vom 18. März 1999 ist abgedruckt bei: Jur.C.S.J. (Pierre Tapia) 1999, Nr. 3, 143-169. 
des Konsultationsreferendums entschied die CSJ im zweiten Urteil vom 19. Januar 1999, dass ,die Volksbefragung entscheidender Faktor dafür ist, dass die zuständigen Organe der Staatsgewalten dazu berufen sind, Mechanismen zur Einberufung und Durchführung einer ANC zu entwerfen, oder das Initiativrecht für eine Änderung oder Reform der Verfassung zu ergreifen“ “20. Die höchstrichterlichen Ausführungen lassen den Rückschluss zu, dass, wenn die Volksbefragung gemäß Art. 181 LOSPP von konsultativem Charakter ist und ein weiteres Tätigwerden der zuständigen Organe ${ }^{21}$ erforderlich macht, sie nicht gleichzeitig Grundlage für die Einberufung einer Verfassunggebenden Versammlung sein kann. Das Ergebnis des Referendums entfaltet demnach Bindungswirkung lediglich im Hinblick auf ein weiteres Tätigwerden der zuständigen Organe. Dies entspricht der Natur eines konsultativen Referendums als demoskopisches Instrument, dessen Ergebnis keine rechtliche Bindungswirkung entfaltet, wenngleich seine mögliche faktische politische Bindungswirkung außer Frage steht.

Die CSJ hatte mit den beiden Urteilen vom 19. Januar 1999 zwar die Verfassungsmäßigkeit eines Konsultationsreferendums über die Einberufung einer ANC festgestellt, nicht aber die Verfassungsmäßigkeit der Einberufung einer ANC auf der Grundlage eines Referendums selbst legitimiert. Mit anderen Worten hatte die CSJ zwar den Weg für eine dritte damals in der Verfassung nicht vorgesehene Möglichkeit der Verfassungsreform geebnet, ohne aber zu der verfassungspolitisch brisanten Frage Stellung zu nehmen, ob eine Verfassunggebende Nationalversammlung durch ein Konsultationsreferendum ohne vorherige Verfassungsreform wirksam einberufen werden könne.

Infolge der in der Bevölkerung vorherrschenden Bereitschaft zum politischen Wandel wurden die Urteile vom 19. Januar 1999 dahingehend ausgelegt, dass eine ANC durch den Willen des Volkes auch ohne vorangehende Verfassungsreform einberufen werden könne $^{22}$. Die Venezolaner hatten das Vertrauen in die staatlichen Institutionen auf der Basis der Verfassung von 1961 verloren und erblickten im Erlass einer neuen Verfassung und der umfassenden Erneuerung von Staatsorganisation und Rechtsordnung den lang ersehnten Ausweg aus der Krise. Eine der ersten Amtshandlungen von Präsident Chávez bestand daher darin die Durchführung eines Konsultationsreferendums über die Einberu-

Urteil Nr. 18 der CSJ/Sala Político-Administrativa vom 19. Januar 1999.

Welches die zuständigen Organe für das Entwerfen der Mechanismen zur Einberufung und Durchführung einer ANC sein sollten, wurde in dem Urteil nicht präzisiert. Zu beachten ist jedoch, dass keinem Organ die dafür erforderliche Zuständigkeit zukam, denn die Möglichkeiten zur Veränderung des Verfassungstextes erschöpften sich in denen der Verfassungsänderung und reform nach Art. 245f. CN (1961).

Siehe dazu: Brewer-Carías, S. 24 und Norbert Lösing, Die Verfassungsgerichtsbarkeit in Lateinamerika, Baden-Baden 2001, S. 405 m.w.N. 
fung einer ANC zu dekretieren ${ }^{23}$. Auf der Grundlage des Dekrets vom 2. Februar 1999 setzte der Nationale Wahlrat (Consejo Nacional Electoral - CNE) per Resolution vom 17. Februar 1999 das Referendum für den 25. April 1999 an und veröffentlichte die im Dekret enthaltenen Grundsätze zur Einberufung der ANC (bases comiciales) ${ }^{24}$. Aufgrund einer Flut von anhängigen Gerichtsverfahren gegen das Dekret und die Resolution und einer kritischen Stellungnahme des Ministerrats änderte Präsident Chávez das Dekret am 10. März $1999 \mathrm{ab}^{25}$. Die sich hieraus ergebenden ,,neuen“ geänderten bases comiciales des Dekrets vom 2. Februar 1999 enthielten neben den Grundsätzen zur Einberufung einer ANC auch Regelungen betreffend deren Aufgabe und Handlungsspielrahmen. Bevor der CNE per Resolution die ,neuen“ bases comiciales erlassen konnte, kassierte der Oberste Gerichtshof am 18. März 1999 eine Passage der Resolution des CNE vom 17. Februar 1999, wonach der Präsident dazu ermächtigt gewesen wäre, nach Abschluss des Referendums die Verfahrensweise zur Nominierung und Wahl der Delegierten der ANC festzulegen $^{26}$. Der CNE veröffentlichte am 23. März 1999 mit der Resolution Nr. 990323-70 die „neuen“ bases comiciales ${ }^{27}$. Am selben und darauffolgenden Tag legte der CNE mit zwei weiteren Resolutionen die genannten Grundsätze fest (Nr. 990323-71) und veröffentlichte die zwei Fragen, die Gegenstand des Referendums sein sollten (Nr. 990323-72) ${ }^{28}$. Die Resolution Nr. 990323-71 enthielt eine Passage, wonach ,sich die ANC als originäre Gewalt, welche die Souveränität des Volkes aufnimmt, ihr eigenes Statut diktieren muss“. Diese Passage stand im Widerspruch mit den im Urteil des Obersten Gerichthofs vom 18. März 1999 aufgestellten Kriterien und Prinzipien und wurde mit Urteil vom 13. April 1999 wegen Verfassungswidrigkeit aufgehoben ${ }^{29}$. Die endgültigen bases comiciales wurden am 21. April 1999 veröffentlicht ${ }^{30}$.

Das Dekret Nr. 3 vom 2. Februar 1999 wurde veröffentlicht in der Gaceta Oficial Nr. 36.634 gleichen Datums. Zum Dekret und den weiteren Rechtsgrundlagen für die Einberufung der ANC siehe: Brewer-Carías, S. 22-30; Lösing, op. cit. (Fn. 22) S. 405ff.; <http://www.analitica.com/ bitblioteca/venezuela/decreto_referendum.asp $>$.

Die Resolution Nr. 990217-32 des CNE vom 17. Februar 1999 findet sich unter: <http://www. badellgrau.com/constresolu.html> .

Der Verwaltungsakt wurde als „Orden“ bezeichnet und in der Gaceta Oficial Nr. 36.658 vom 10. März 1999 veröffentlicht. Urteil Nr. 271 der CSJ/Sala Político-Administrativa vom 18. März 1999, op. cit. (Fn. 19).

Die Resolution Nr. 990323-70 des CNE wurde veröffentlicht in der Gaceta Oficial Nr. 36.669 vom 25. März 1999.

Die Resolution Nr. 990323-71 des CNE wurde veröffentlicht in der Gaceta Oficial Nr. 36.669 vom 25. März 1999, die Resolution Nr. 990323-72 in der Gaceta Oficial Nr. 36.672 vom 30. März 1999. worden ist, ist abgedruckt bei: Jur.C.S.J. (Pierre Tapia) 1999, Nr. 3, 362-373 und findet sich unter: <http://www.analitica.com/bitblioteca/csj/15679-311.asp>. 
Das venezolanische Volk stimmte am 25. April 1999 im Rahmen eines landesweiten Referendums mit $88 \%$ (bei einer Wahlbeteiligung von 39,1\%) für die Einberufung einer $\mathrm{ANC}^{31}$. Mit Urteil vom 3. Juni 1999 stellte das Oberste Gericht (CSJ) fest, dass sich die Wählerschaft für die Einberufung einer ANC und die Annahme der bases comiciales zur Regelung der ANC ausgesprochen hatte und entschied, dass sich der Ausdruck des Volkswillens in eine obligatorisch zu erfüllende Entscheidung gewandelt hatte, die höchsten Rang besitzt ${ }^{32}$. Bereits kurz vor der Wahl zur ANC entschied die CSJ mit Urteil vom 21. Juli 1999, dass der verfassunggebende Prozess de iure gewesen sei, der im Rahmen des geltenden Rechts vollzogen wurde ${ }^{33}$.

\section{b) Entwurf und In-Kraft-Treten der neuen Verfassung}

Aus der Wahl zur ANC am 25. Juli 1999 ging aufgrund der damaligen Popularität von Chávez erwartungsgemäß eine von seinen Anhängern dominierte Verfassunggebende Nationalversammlung hervor (125 der 131 Sitze) ${ }^{34}$. Die ANC trat Anfang August erstmals zusammen, gab sich ein Statut und nahm ihre Tätigkeit auf. Binnen kürzester Zeit entwarf sie eine neue Verfassung, die durch ein Referendum am 15. Dezember 1999 mit einer Mehrheit von $71 \%$ (bei einer Wahlbeteiligung von $46 \%$ ) angenommen wurde ${ }^{35}$. Mit ihrer Veröffentlichung trat die sechsundzwanzigste Verfassung des Landes in $\mathrm{Kraft}^{36}$. Deren zentrale Abweichungen gegenüber der vorherigen Verfassung lassen sich wie folgt zusammenfassen:

Siehe: Peter Rösler, Lateinamerika Jb. (2000) 194, 197; <http://www.georgetown.edu/pdba/ Elecdata/Venezuela/ref99.html > und <http://www.analitica.com/bitblioteca/anc/default.asp >.

Die zitierte Passage des Urteils vom 3. Juni 1999 ist abgedruckt bei: Jur.C.S.J. (Pierre Tapia) 1999, Nr. 5, 229 und findet sich unter: <http://www.analitica.com/bitblioteca/csj/regimen_constituyente.asp>.

Das Urteil Nr. 942 der CSJ/Sala Político-Administrativa vom 21. Juli 1999 ist abgedruckt bei: Jur.C.S.J. (Pierre Tapia) 1999, Nr. 6, 195-202. Siehe auch: Brewer-Carías, S. 29; Lösing, op. cit. (Fn. 22) S. 408.

Brewer-Carías, S. 30. Im Ergebnis leicht abweichend (122 der 131 Sitze) Neuman/McCoy, S. 26. Unter anderen waren die First Lady Marisabel de Chávez, der Bruder des Präsidenten Adán Chávez. Frías und Luis Miquilena, Delegierte der ANC, vgl.: <http://www.analitica.com/ constituyente/asambleistas/> und <http://www.analitica.com/bitblioteca/anc/constituyentes.asp $>$.

Eingehend: Neuman/McCoy, S. 33-38. Siehe auch: <http://www.georgetown.edu/pdba/Elecdata/ Venezuela/venref.html> und <http://www.analitica.com/bitblioteca/anc/default.asp >.

Die Constitución de la República Bolivariana de Venezuela (CRBV) von 1999 wurde veröffentlicht in der Gaceta Oficial Nr. 36.860 vom 30. Dezember 1999. Auf der korrigierten Fassung, die nicht Gegenstand des Referendums gewesen ist und in der Gaceta Oficial Nr. 5453 Extraordinario vom 24. März 2000 veröffentlicht wurde, basieren die weiteren Ausführungen. Der Verfassungstext ist abrufbar unter: <http://www.georgetown.edu/pdba/Constitutions/Venezuela/ven1999.html>. 
- In Anlehnung an den Freiheitshelden Simón Bolívar wird das Land in República Bolivariana de Venezuela umbenannt (Art. 1) ${ }^{37}$.

- Erweiterung der Kompetenzen des Staatspräsidenten. Seine Amtszeit wird von 5 auf 6 Jahre verlängert und gleichzeitig die Möglichkeit einer direkten Wiederwahl eingeführt (Art. 230) ${ }^{38}$. Das Amt des Geschäftsführenden Vize-Präsidenten wird geschaffen (Art. 238 ff. ${ }^{39}$, der neben den Ministern vom Präsidenten zu ernennen ist (Art. 236 Nr. 3) ${ }^{40}$. Der Präsident hat nunmehr das alleinige Recht, das Parlament aufzulösen (Art. 236 Nr. $21)^{41}$ und Offiziere vom Rang des Oberst an direkt zu befördern (Art. 236 Nr. 6) ${ }^{42}$.

- Umwandlung des Zwei-Kammern-Kongresses in eine Nationalversammlung (Asamblea Nacional) mit nur einer Kammer (Art. 186 ff.) ${ }^{43}$. Die Reduzierung auf eine Kammer bedeutet für die Bundesstaaten den Verlust einer bedeutenden Vertretungsstufe auf Bundesebene $^{44}$ und eine geringere Einbindung in das Gesetzgebungsverfahren ${ }^{45}$. Der zum Ausgleich geschaffene Consejo Federal de Gobierno nimmt lediglich beratende Funktionen wahr (Art. 185).

- Schaffung zweier neuen Staatsgewalten: Die Bürgergewalt, der Poder Ciudadano (Art. 273 ff.) ${ }^{46}$ und die Wählergewalt, der Poder Electoral (Art. 292 ff.) ${ }^{47}$. aufsichtsbehörde ausgeübt, der aus fünf Mitgliedern besteht. Aufgabe dieser Staatsgewalt ist die Reglementierung, Durchführung sowie Koordination aller Wahlen politischen Charakters. Siehe zum Poder Electoral: Motive, Gaceta Legal (2000) 318, 327 und Brewer-Carías, S. $145 f$. 
- Einführung von zwei neuen Kammern im Obersten Gericht (TSJ) aufgrund der zwei neu geschaffenen Gewalten. Verlängerung der Amtszeit der Richter am Obersten Gericht von 9 auf 12 Jahre (Art. 264) ${ }^{48}$.

- Verankerung des Prinzips der Direktdemokratie: Konsultationsreferenda können über Fragen nationalen Interesses einberufen werden (Art. 71). Ebenso besteht die Möglichkeit, alle gewählten Amtsträger bis hin zum Staatspräsidenten ihres Amtes zu entheben (referendo revocatorio) (Art. 72$)^{49}$. Über Gesetzesinitiativen (referendo aprobatorio) sowie über die Abschaffung von Gesetzen (referendo abrogatorio) kann im Wege der Volksabstimmung entschieden werden (Art. 73f.).

- Einführung des Wahlrechts für Angehörige des Militärs (Art. 330).

- Einführung des Rechts, jedwedem Regime, Gesetzgebung oder Autorität die Anerkennung zu versagen, das sich demokratischen Werten, Prinzipien und Garantien entgegenstellt oder Menschenrechte verletzt (Art. 350) - die Desobediencia Civil ${ }^{50}$.

Die von Chávez für gescheitert erklärte repräsentative Demokratie war einer direkten partizi pativen Demokratie gewichen. Erklärte Absicht des Präsidenten war es, die Regierung dem Volke näher zu bringen und die Menschen in Venezuela verstärkt am politischen Prozess teilhaben zu lassen. Zudem sollten die Staatsgewalten einer stärkeren Kontrolle durch das Volk unterworfen sein. Entsprechende Kontroll- und Teilhabemechanismen wurden in Gestalt der Wählergewalt und der zahlreichen plebiszitären Elemente in die neue Verfas sung aufgenommen. Die durch diese Elemente ermöglichte Mitwirkung der Bürger am politischen Prozess diente zugleich der demokratischen Sicherung der Macht von Präsident Chávez.

Die erste Frage des Konsultationsreferendums vom 25. April 1999 lautete: „Berufen Sie eine Verfassunggebende Nationalversammlung mit dem Vorhaben ein, den Staat umzuformen und eine neue Rechtsordnung zu schaffen, die das effektive Funktionieren einer sozialen und partizipativen Demokratie ermöglicht?“. Hieraus leitete die ANC die Ermächtigung ab, auch losgelöst von der Redaktion einer neuen Verfassung, den Staat umzuwandeln und eine neue Rechtsordnung zu schaffen. Die ANC gab sich im August 1999 ein Statut

48

Auch der Oberste Gerichtshof kann den Präsidenten des Amtes entheben, Art. 233, 266 Nr. 2 CRBV.

50

Hintergrund für die Einführung dieses Widerstandsrechts gegen ein Unrechtsregime, ist der Versuch einer Rechtfertigung für den von Hugo Chávez angeführten Putschversuch im Jahre 1992. Chávez berief sich damals auf Art. 132 CN (1961). 
und erklärte sich darin zum originären Obersten Staatsorgan ${ }^{51}$. Nach Art. 1 ihres Statuts ermächtigte sie sich dazu, über Umfang und Dauer der Aktivitäten der Staatsgewalten zu entscheiden. Alle Organismen der Öffentlichen Gewalt wurden der ANC untergeordnet und dazu verpflichtet, die von ihr stammenden Rechtsakte zu erfüllen. Das Statut bestimmte weiter, dass die geltende Verfassung von 1961 und die gesamte Rechtsordnung in Kraft bliebe, solange sie nicht im Widerspruch zu den Rechtsakten und Entscheidungen der ANC stehe.

In Wahrnehmung dieser Befugnisse dekretierte die ANC am 12. August 1999 die Reorganisation aller Organe der Öffentlichen Gewalt ${ }^{52}$. Am 19. August dekretierte sie den Notstand über das Justizwesen, die Reorganisation der Judikative und die Einberufung der Judikativen Notstandskommission, welche die regulären Organe der Rechtsprechung ersetzte $^{53}$. Die Comisión de Emergencia Judicial bestand aus neun Mitgliedern, von denen zuvor vier der ANC angehört hatten. Die übrigen fünf Mitglieder waren von ihr ernannt worden. Die Aufgabe der Kommission bestand neben der Untersuchung des gesamten Justizwesens auf Fälle von Korruption und Amtsmissbrauch in der Erarbeitung von Vorschlägen zur Reorganisation des Justizwesens und ihrer Durchsetzung nach deren Annahme durch die ANC. In der Folgezeit wurden zahlreiche Richter wegen Korruptionsvorwürfen entlassen oder suspendiert. Die Neueinstellungen fielen in den Zuständigkeitsbereich der Notstandskommission.

Am 25. August 1999 erließ die ANC ein Dekret zur Regulierung der Aufgaben der Legislative. Hierdurch wurden alle ordentlichen und außerordentlichen Kongresssitzungen aufgehoben $^{54}$. Des Weiteren wurde die Ausübung der Aufgaben des Kongresses mehrheitlich auf die Gesetzgebungskommission der ANC sowie eine Comisión Delegada übertragen. Die Comisión Legislativa de la Asamblea Nacional Constituyente bestand aus sieben Mitgliedern, von denen zuvor vier der ANC angehört hatten. Die innenpolitische Lage spitzte sich zu. Nachdem die Kirche zwischen der ANC und dem Kongress vermittelt hatte, kam am 9. September 1999 eine Vereinbarung zustande, wonach der Kongress bis Ende

Brewer-Carías, S. 31f. m.w.N.; Lösing, op. cit. (Fn. 22) S. 409; Neuman/McCoy, S. 30; Rösler, op. cit. (Fn. 7) 199. Das Statut wurde veröffentlicht in der Gaceta Oficial Nr. 36.786 vom 14. September 1999.

Das Dekret wurde veröffentlicht in der Gaceta Oficial Nr. 36.764 vom 13. August 1999.

Das Dekret wurde veröffentlicht in der Gaceta Oficial Nr. 36. 782 vom 8. September 1999.

54

Das Dekret vom 25. August 1999 wurde veröffentlicht in der Gaceta Oficial Nr. 36. 772 vom 26. August 1999. Das Dekret wurde am 30. August 1999 reformiert und in der Gaceta Oficial Nr. 36. 776 vom 31. August 1999 veröffentlicht. 
des Jahres seine Tätigkeit wieder aufzunehmen hatte ${ }^{55}$. Die wichtigen Beschlüsse des Kongresses blieben jedoch weiterhin der Bestätigung durch die ANC unterworfen.

\section{2. $\quad$ Etablierung des Übergangsregimes}

Mit dem In-Kraft-Treten der neuen Verfassung Ende 1999 war die Besetzung der neu geschaffenen Ämter und die Relegitimation aller bereits bestehenden politischen Wahl ämter notwendig geworden. Die zu diesem Zweck durchzuführenden „Megawahlen“ umfassten neben den Präsidentschafts-, Parlaments-, Gouverneurs- und Kommunalwahlen auch die Wahl der Repräsentanten für das Anden- und das Lateinamerikanische Parla ment ${ }^{56}$. Die ANC dekretierte am 22. Dezember 1999 einen Zwischenzustand (Régimen de Transición del Poder Público ${ }^{57}$, der die demokratisch gewählten Organe und Staatsgewalten auflöste und ein Übergangsregime etablierte, um das Vakuum zwischen dem Ende des Mandats der $\mathrm{ANC}^{58}$ und den Neuwahlen auszufüllen ${ }^{59}$. Der renommierte venezolanische Verfassungsrechtler Brewer-Carías wies darauf hin, dass die ANC dieses Vakuum erst durch besagtes Dekret herbeigeführt hatte, da es die demokratisch gewählten Organe und Staatsgewalten aufgelöst hatte. Durch das Dekret vom 22. Dezember 1999 wurde der Kongress endgültig aufgelöst, (Art. 4) und dessen Aufgaben vom 1. Februar 1999 an und bis zur ersten Sitzung der neu zu wählenden Nationalversammlung (Parlament) einer Nationalen Gesetzgebenden Kom mission, dem sogenannten Congresillo ${ }^{60}$, übertragen (Art. 5-9). Die vom venezolanischen Volk demokratisch gewählten Repräsentanten wurden somit von Personen ersetzt, die von der ANC bestimmt waren. Auf der Ebene der Bundesstaaten und der Gemeinden wurde in entsprechender Weise verfügt (Art. 11-15). Die CSJ wurde aufgelöst und deren Kammern im neu geschaffenen TSJ integriert (Art. 17). Neben den neuen Richtern ernannte die ANC in diesem Dekret auch den Ombudsmann (Defensor del Pueblo), den Oberstaatsanwalt, den Obersten Rechnungsprüfer und die Mitglieder des Nationalen Wahlrats (Art. 19, 34-36, 40).

Siehe: Brewer-Carías, S. 33 mit Verweis auf den Abdruck der Vereinbarung in der Tageszeitung El Nacional vom 10. September 1999, S. D-4; Rösler, op. cit. (Fn. 31) 194, $202 \mathrm{f}$.

Die zu wählenden Posten sind aufgelistet im Dokument der OAS: OEA/Ser.D/XX/SG/UPD/II.30, S. 24, <http://www.upd.oas.org/lab/Documents/publications/electoral_observation/2000/pbl_30_ 2000_spa.pdf.>. Der Begriff der „Megawahlen“ geht zurück auf das Regierungsprogramm von Hugo Chávez.

Das Dekret wurde veröffentlicht in der Gaceta Oficial Nr. 36.859 vom 29. Dezember 1999.

Ende Januar 2000 löste sich die Verfassunggebende Nationalversammlung (ANC) auf.

59

Siehe hierzu das Regierungsprogramm: <http://www.mpd.gov.ve/prog-gob/prog_gob2/e_politico. $\mathrm{htm}>$.

60

Ein Gremium, das sich unter der Leitung von Luis Miquilena - dem ehemaligen Präsidenten der ANC - konstituierte und dessen insgesamt 21 Mitglieder mehrheitlich zuvor der ANC angehört hatten. 


\section{Institutionalisierung der neuen Republik}

Mit der Organisation und Durchführung der „Megawahlen“ wurde der Nationale Wahlrat (CNE) beauftragt. Die ANC dekretierte am 30. Januar 2000 den Estatuto Electoral del Poder Público und setzte die Durchführung der Wahlen für den 28. Mai $2000 \mathrm{an}^{61}$. Neben Korruptionsskandalen ${ }^{62}$ prägten die mit dem erstmaligen Einsatz eines elektronischen Abstimmungs- und Zählsystems verbundenen Probleme die Zeit vor den Wahlen. Der Oberste Gerichtshof verschob am 25. Mai 2000 die Wahlen wegen mangelhafter Informationsmöglichkeiten über die Kandidaten und schwerer technischer Mängel ${ }^{63}$. Die Mitglieder des CNE traten zurück. Am 3. Juni ernannte der Congresillo bis zum Erlass des neuen Gesetzes über die Wählergewalt und der endgültigen Wahlen die insgesamt 15 Mitglieder (5 Mitglieder und 10 Stellvertreter) des $\mathrm{CNE}^{64}$.

In der „Megawahl“65 vom 30. Juli 2000 wurde Chávez als Staatsoberhaupt und Regierungschef mit 59,5\% (bei einer Wahlbeteiligung von 56,5\%) eindrucksvoll bestätigt. In den Parlamentswahlen erzielten die Chávez unterstützenden Regierungsparteien 99 der insgesamt 165 Sitze. Die Altparteien AD und COPEI erzielten gemeinsam lediglich 38 Sitze. Die Regierungsparteien stellten mit 14 Gouverneuren deren Mehrzahl. AD und COPEI stellten gemeinsam lediglich 4 Gouverneure.

61

62

63

64

65

Siehe: Gaceta Oficial Nr. 36. 884 vom 2. Februar 2000.

Siehe hierzu den Bericht des Journalisten Augustín Beroes: La corrupción en tiempo de Chávez, unter: <http://es.geocities.com/malversacion>.

Siehe das Urteil Nr. 483 des TSJ/Sala Constitucional vom 25. Mai 2000, vgl. <http://www. analitica.com/bitblioteca/csj/megaelecciones2.asp\#sesion>. Siehe auch das Urteil Nr. 811 des TSJ/Sala Constitucional vom 26. Juli 2000 und Neuman/McCoy, S. 55, die irrtümlich den TSJ als CSJ bezeichnen. Die Urteile des TSJ sind abrufbar unter: <http://www.tsj.gov.ve>.

Siehe: Gaceta Oficial Nr. 36.965 vom 5. Juni 2000. Das Gesetz (Ley Orgánica del Poder Electoral - LOPE) wurde im November 2002 erlassen und in der Gaceta Oficial Nr. 37.573 vom 19. November 2002 veröffentlicht. Die Mitglieder wurden im August 2003 vom TSJ statt vom Parlament vorläufig ernannt (Urteil Nr. 2341 des TSJ/Sala Constitucional vom 25. August 2003). Trotz des Urteils des TSJ, nach Ablauf einer zehntägigen Frist ab Urteilsverkündung, selbst binnen zehn Tagen die Ernennung vorzunehmen, konnten sich die politischen Kräfte im Parlament nicht über die Besetzung des CNE einigen (Urteil Nr. 2073 des TSJ/Sala Constitucional vom 4. August 2003, und Urteil Nr. 1582 des TSJ/Sala Constitucional vom 12. Juni 2003). Das Verfahren im Parlament war dadurch erschwert worden, dass zur Wahl der Mitglieder eine ZweiDrittel-Mehrheit erforderlich ist, Art. 8 LOPE.

Eingehend zur „Megawahl“: Neuman/McCoy und OEA/Ser.D/XX/SG/UPD/II.30 vom 14. Dezember 2000, <http://www.upd.oas.org/lab/Documents/publications/electoral_observation/2000/pbl_ 30_2000_spa.pdf.>. Siehe auch: Peter Rösler, Lateinamerika Jb. (2001) 220, 226; Friedrich Welsch / Nikolaus Werz, Staatsstreich gegen Chávez: Institut für Iberoamerika-Kunde 2000, 205215 (Brennpunkt Lateinamerika, Nr. 20-00); <http://www.electionworld.org/election/venezuela. htm>. 


\section{Durchführung des Regierungsprogramms}

Nach den erfolgten „Megawahlen“ galt es, die zur Institutionalisierung der neuen Republik und zur Durchsetzung des Regierungsprogramms notwendigen Gesetze zu erlassen. Venezuela wurde einer umfassenden Rechtsreform unterzogen. Seit dem Regierungsantritt von Präsident Chávez sind bis Ende Dezember 2002 insgesamt 211 neue Gesetze in Kraft getreten, von denen $49 \%$ Gesetzesdekrete (decretos leyes) waren, die der Präsident auf der Grundlage zweier Ermächtigungsgesetze erließ (Ley habilitante) ${ }^{66}$. Die Reform weist einen Mangel an geraden Linien und Qualität auf. Viele Gesetze wurden kurzfristig reformiert und mussten aufgrund formeller Fehler wiederholt veröffentlicht werden. Oft wurde nicht einmal ein Minimum an gesellschaftlichem Konsens erreicht. Die neuen Gesetze wurden gleichermaßen von den betroffenen Interessengruppen stark kritisiert.

Hugo Chávez hatte die Abkehr vom neoliberalen Wirtschaftskurs der korrupten Parteien oligarchie des Landes angekündigt und für die venezolanische Wirtschaft einen dritten Weg zwischen Kommunismus und inhumaner Marktwirtschaft propagiert ${ }^{67}$. Zur Steigerung der wirtschaftlichen Produktivität sollte insbesondere die monoproduktive Konzentration auf den Erdölsektor überwunden werden. Gleichzeitig versprach Chávez, die unteren Sozialschichten am Erlös der Ölindustrie zu beteiligen. Trotz dynamisch wirkenden Starts ist die Bilanz von Chávez' Wirtschaftspolitik desolat. Die unvermindert fortschreitende Rezession ist augenfällig - auch ohne die Auswirkungen des Generalstreiks. Folgende wirtschaftliche Indikatoren und Prognosen bestätigen dies ${ }^{68}$ :

\begin{tabular}{|lcccc|}
\hline Veränderung des Bruttoinlandprodukts & $(2000)$ & $(2001)$ & $(2002)$ & $(2003 \mathrm{p})$ - \\
(real): & $3,2 \%$ & $2,8 \%$ & $-8,9 \%$ & $13,5 \%$ \\
Inflationsquote: & $13,4 \%$ & $12,3 \%$ & $22,4 \%$ & $32,8 \%$ \\
Arbeitslosenquote: & $13,2 \%$ & $12,8 \%$ & $16,6 \%$ & $21 \%{ }^{69}$ \\
Wechselkurs (Bolívar je US\$): & 670 & 763 & 1.500 & $1.600^{70}$ \\
\hline
\end{tabular}

66

Das erste Ermächtigungsgesetz ist die Ley Orgánica que autoriza al Presidente de la República para dictar Medidas Extraordinarias en materia económica y financiera requeridas por el interés público, veröffentlicht in der Gaceta Oficial Nr. 36.868 vom 26. April 1999. Das zweite Ermächtigungsgesetz ist die Ley que autoriza al Presidente de la República para dictar Decretos con Fuerza de Ley en las materias que se delegan, veröffentlicht in der Gaceta Oficial Nr. 37.076 vom 13. November 2000.

67

68

69

Siehe hierzu das Regierungsprogramm: <http://www.mpd.gov.ve/prog-gob/pg_eqeco.htm>.

Quellen: Banco Central de Venezuela (BCV), Ministerio de Finanzas, Instituto Nacional de Estadísticas (INE), Comisión Económica para América Latina y el Caribe (CEPAL), Centro de Estudios Latinoamericanos (CESLA), Datanalisis, Bundesagentur für Aussenwirtschaft (bfai), Dresdner Bank und Dresdner Bank Lateinamerika (Stand: Juli 2003).

Weniger als 50\% der Bevölkerung (ca. 24. Mio.) ist erwerbstätig. Nahezu 50\% der Beschäftigten ist im informellen Sektor tätig. 
Die Regierung erreichte die selbstgesteckten wirtschaftlichen Ziele nicht. Einer der Gründe hierfür mag in der Konzentration auf staatsorganisatorische und innenpolitische Strukturumwälzungen liegen. Auch mag ein Zeitraum von vier Jahren nicht sehr aussagekräftig sein. Dennoch wurden dringend notwendige grundlegende Strukturreformen in der Wirtschaft und im Staatsapparat nicht eingeleitet. Eine zukunftsorientierte Wirtschafts- und Finanzpolitik der Regierung Chávez ist nicht zu erkennen. Vielmehr entsteht der Eindruck, dass sich die inkohärente Politik seiner diskre ditierten Vorgänger AD und COPEI fortsetzt.

Hugo Chávez' erklärte Absicht war die Herstellung sozialer Gerechtigkeit ${ }^{71}$. Armut, Arbeitslosigkeit und Kriminalität wollte er bekämpfen, marginalisierte Schichten und indi gene Völker integrieren, das soziale Netz ebenso wie das Bildungs- und Gesundheitswesen ausbauen. Nennenswert verbesserten sich die Lebensbedingungen der armen Bevölke rungsschichten indessen nicht. Rund $71 \%$ der Bevölkerung lebt in Armut, hier von sind wiederum circa $36 \%$ extrem $\operatorname{arm}^{72}$. Die negative Kriminalitätsstatistik belegt, wie die persönliche Sicherheit schwindet. Im ersten Halbjahr 2002 erhöhten sich im Ver hältnis zu den Vorjahresdaten die Straftaten um $44 \%$ auf circa 124.000 Straftaten $^{73}$. Die zahlreichen Interimsrichter erschweren eine effektive Strafverfolgung. Es entsteht ein strafloser $\operatorname{Raum}^{74}$.

Die Regierung führte am 6. Februar 2003 Devisen- und Preiskontrollen ein (1.600 Bs. je US\$), vgl. den Convenio Cambiario Nr. 1 und 2 zwischen dem Finanzministerium und dem Banco Central de Venezuela, veröffentlicht in der Gaceta Oficial Nr. 37.625 vom 5. Februar 2003. Durch das Dekret Nr. 2.302 vom 5. Februar 2003 wurde die Comisión de Administración de Divisas (CADIVI) geschaffen und durch das Dekret Nr. 2.303 vom selben Tag personell besetzt, beide Dekrete finden sich in der oben genannten Gaceta Oficial. Auf dem Schwarzmarkt wurde der US\$ für bis zu 2.200 Bs. gehandelt. Aufgrund der Devaluation des Bolívar steigt die Inflation weiter an, zugleich ermöglicht sie es der Regierung ihr Steuerdefizit zu reduzieren. Nach Presseangaben hatte Chávez angekündigt, es werde keinen einzigen Dollar für die Putschisten und Unruhestifter geben und damit den politischen Hintergründe dieser Maßnahme als Druckmittel gegen die Opposition deutlich werden lassen, siehe: Venezuela verhängt Preis- und Devisenkontrollen, FAZ vom 7. Februar 2003.

Siehe dazu das Regierungsprogramm: <http://www.mpg.gov.ve/prog-gob/pg_eqsol-htm>.

Departamento de Investigaciones Económicas del IIES-UCAB. Siehe auch: <http://www.who.int/ whr/2002/annex/en/>.

Siehe: <http://www.venezuelalibre.net/noticias914.20020918.html>; <http://www.iadb.org/exr/ doc98/pro/Pve0115.pdf>; <http://universal.eud.com/2003/01/04/04280AA.shtml>. Siehe auch den Bericht des Programa de Naciones Unidas para el Desarrollo (PNUD): <http://www. pnud.org.ve/IDH97/violenciacodh.htm>.

74 Die Interamerikanische Menschenrechtskommission (IACHR) zeigt sich besorgt aufgrund der Tatsache, dass drei Jahre nach Beginn der Reorganisation der Judikative noch immer weit über $70 \%$ der Richter Interimrichter sind, siehe: <http://www.cidh.org/comunicados/spanish/2003/ 5.03.htm> und <http://www.cidh.org/annualrep/2002sp/cap.4d.htm>. 
Die Erschließung und Konsolidierung des Staatsgebietes umschloss die Förderung der Landwirtschaft, den Ausbau der Infrastruktur und die Förderung dezentralisierender Kräfte, wie es der Tourismussektor und die klein- und mittelständischen Betriebe sind ${ }^{75}$. Auch auf diesem Gebiet hatte die Regierung keinen durchschlagenden Erfolg. Die Durchführung des Plan Bolívar 2000, ein zivil-militärisches Projekt, das in drei Stufen die Zusammenarbeit von Zivilgesellschaft und Militär zur Durchführung von Entwicklungsprogrammen unter anderem im Bereich des Sozial- und Bildungssystems, der Infrastruktur und der Landwirt schaft koordiniert, konnte nichts maßgeblich verbessern ${ }^{76}$.

Im Hinblick auf die Souveränität und die multipolare Integration sah das Konzept der Außenpolitik laut Regierungsprogramm eine enge Bindung an lateinamerikanische Staaten und eine strategische Bindung an die USA, EU und Asien vor ${ }^{77}$. Heute verbindet die Außenpolitik von Hugo Chávez Venezuela insbesondere mit dem Chávez-Idol Fidel Castro. Vorwürfe, wonach die Regierung der kolumbianischen FARC und ELN Guerrilla ${ }^{78}$ nahe steht, konnten nicht widerlegt werden. Der Kurs ist deutlich anti-amerikanisch und gegen die Freihandelszone (FTAA) gerichtet.

\section{Gescheiterter Staatsstreich}

Wie sehr Chávez daran gelegen ist, seine Macht im Bereich der Wirtschaft auszuweiten, wurde deutlich, als er im Februar 2002 seine Absicht erklärte, Kontrolle über die PDVSA ausüben zu wollen. Damit begann ein erbitterter Kampf um die wichtigste Geldquelle des Landes ${ }^{79}$, der vorläufig im gescheiterten Staatsstreich gipfelte. Die Erdölförderung stellt $25 \%$ des Bruttoinlandsproduktes, $50 \%$ der Staatseinnahmen und $80 \%$ der Exporteinnahmen dar. Während der Fernseh- und Radiosendung Aló Presidente vom 7. April 2002 entließ Chávez medienwirksam den gesamten Vorstand der PDVSA und besetzte die Posten mit regierungstreuen Anhängern. Zuvor hatte der Vorstand wegen der das Unternehmen betreffenden Regierungspolitik zum Streik aufgerufen. Der Gewerkschaftsdachverband (CTV) mit engen Beziehungen zum traditionellen politischen System, rief zwei Tage später zum Generalstreik auf, dem sich der Unternehmerdachverband (Fedecámaras)

Siehe: <http://www.mpd.gov.ve/prog-gob/pg_eqter.htm>.

Siehe zum Plan Bolívar 2000: <http://www.mpd.gov.ve/prog-gob/proyb2000.htm>; <http://www. es.geocities.com/malversacion>.

Siehe: <http://www.mpd.gov.ve/prog-gob/pg_eqmun.htm>.

Fuerzas Armadas Revolucionarias de Colombia (FARC) und Ejército de Liberación Nacional $(\mathrm{ELN})$.

Venezuela zählt mit einer Produktion von durchschnittlich 3 Millionen Fässer am Tag zu den fünf bedeutendsten erdölexportierenden Ländern der Welt. Zudem verfügt das Land über rund 77,7 Milliarden Fässer nachgewiesener Rohölreserven, vgl. hierzu: <http://www.opec.org/>. 
anschloss. Die am 11. April 2002 stattfindende Großdemonstration gegen Chávez, deren ursprüngliches Ziel der Unternehmenssitz der PDVSA gewesen war, setzte sich nach dessen Erreichen weiter in Richtung Präsidentenpalast Miraflores in Bewegung. Dort hatten sich bereits Chávez-Anhänger zum Zeichen der Solidarität mit der Regierung eingefunden. Als die Demonstrationen aufeinander stießen, wurde die Stimmung explosiv. Bald darauf schossen Scharfschützen in die Menge, 17 Demonstranten kamen zu Tode, Hunderte wurden verletzt. Chávez gab den Befehl, Panzer auffahren zu lassen. Ob er auch den Schussbefehl an die Scharfschützen gab, ist nicht mit letzter Sicherheit aufzuklären. Generäle der Guar dia Nacional und weitere Militärs, die mit der Opposition sympathisierten, verweigerten den Befehl und forderten seinen Rücktritt. Chávez wurde inhaftiert und die Putschisten zogen in den Präsidentenpalast ein. Ob Chávez an diesem oder darauffolgenden Tag freiwillig oder unfreiwillig zurücktrat, ist nicht aufzuklären, wird allerdings von der Opposition, wenn auch etwas realitätsfremd, vehement behauptet. Der Oberste Gerichtshof (TSJ) entschied später, keine Anklage gegen die an der Verhaftung des Präsidenten beteiligten Militärs zu erheben ${ }^{80}$. Der damalige Präsident von Fedecámaras, Pedro Carmona Estanga, wurde als Interimpräsident vereidigt. Allerdings kehrte tagsdarauf die Chávez Regierung mit Unterstützung von Teilen des Militärs ${ }^{81}$ und auf Druck der Chávez Anhänger wieder zurück an die Macht, woraufhin Carmona zurücktrat. Vize-Präsident Diosdado Cabello übernahm gemäß Art. 234 CRBV die Amtsgeschäfte und wurde als Interimpräsident vereidigt. Nach nur 48 Stunden war Chávez am 14. April 2002 wieder im Amt.

\section{Kritik der Opposition}

Die von der Opposition gegenüber der Regierung Chávez vorgebrachte Kritik ist facettenreich, und es wäre verfehlt, sie auf die Verteidigung asozialer Interessen der Wohlhabenden zu reduzieren. Die Opposition ist im Bündnis Coordinadora Democrática organisiert und umfasst neben zahlreichen politischen Parteien ${ }^{82}$ den Unternehmerdachverband, den Gewerkschaftsdachverband, das staatliche Erdölunternehmen, die privaten Medien und

Das Urteil Nr. 38 des TSJ/Sala Plena Accidental vom 14. August 2002 findet sich mit Datum vom 19. September 2002 bei <http://www.tsj.gov.ve> und unter <http://www.el-nacional.com/ referencia/documentos/pdf/Ponencia_Franklin_Arrieche.pdf $>$.

Das Fallschirmjäger-Bataillon, die Palastgarde und Einheiten des Militärs im Landesinneren erklärten ihre Loyalität gegenüber Präsident Chávez, vgl. die Dokumentation von Kim Bartley und Donnacha O'Brian: Venezuela. El Presidente - Hugo Chávez. Ausgestrahlt am 29. März 2003 bei dem Fernsehsender arte um 20:45 Uhr.

AD, COPEI, MÁS, Proyecto Venezuela (PV), Primero Justicia (PJ) und Causa Radical (Causa R). Zu beachten ist, dass es sich beim Movimiento al Socialismo (MÁS) um eine ehemalige Regierungspartei unter Chávez handelt. Siehe: <http://www.coordinadorademocratica.com>. 
Teile der Kirche. Auch einstige politische Weggefährte von Hugo Chávez ${ }^{83}$ und weite Teile der Mittelschicht, deren Hoffnungsträger für einen Neuanfang Chávez war, haben resigniert, als den großen Vorhaben kaum Taten folgten. Im April 2002 schlossen sich zudem Teile des Militärs der Opposition an ${ }^{84}$.

\section{Unfähigkeit der Regierung}

In erster Linie zielt die Kritik der Opposition auf die Unfähigkeit der Regierung, trotz der hohen Ölpreise auf dem Weltmarkt, keine Lösungen für die grundlegenden Probleme Venezuelas wie Armut, Arbeitslosigkeit, Rezession, Korruption und Kriminalität anzubieten. Die Regierung von Präsident Chávez hat im Hinblick auf die wirtschaftlichen und sozialpolitischen Probleme wenig bewirkt. Zwar ist das Zweiparteiensystem der Punto-Fijo Parteien AD und COPEI, das das Land durch Fehlplanung und Korruption heruntergewirtschaftet hatte, überwunden und die traditionell herrschenden Parteien diskreditiert und nahezu vom politischen Geschehen abgekoppelt. Eine nachweisbare Verbesserung der Lage Venezuelas ist indes ausgeblieben. Nach wie vor gibt es in Venezuela Korruption, mit der Klientelismus, Vetternwirtschaft, illegale Einnahmen und Ämterpatronage einhergehen ${ }^{85}$. Lediglich die Begünstigten sind andere ${ }^{86}$. Dem Vertrauensverlust der Bevölkerung gegenüber staatlichen Institutionen wurde nicht abgeholfen. Neben der zunehmenden Kapitalflucht ist ein enormer Rückgang von Neuinvestitionen aus dem Aus- und Inland zu verzeichnen. Ferner ist die Abwanderungsquote junger Menschen mit einem hohen Bildungsstand gestiegen.

Beispielsweise Luis Miquilena (zurückgetretener Minister für Justiz und Inneres, ehemaliges Führungsmitglied der Regierungspartei MVR und Präsident der ANC), Alfredo Peña und Francisco Arias Cardenas (Ex-Offizier und Putschist von 1992, zurückgetretener Gouverneur des Bundesstaates Zulia und Herausforderer von Hugo Chávez in der Präsidentschaftswahl 2000).

Teile des Militärs waren auf der Plaza Francia in Caracas versammelt und forderten ebenfalls den Rücktritt von Präsident Chávez, vgl. die Website der Militärs: <http://www.militaresdemocraticos. com>.

Angaben von Transparency International (Oktober 2003) zu Folge, erhält Venezuela im Corruption Perceptions Index (CPI) einen Wert von 2.4 Punkten auf der Skala (10 Punkte für Länder ohne Korruption, 0 Punkte für Länder mit der höchsten Wahrnehmung von Korruption), vgl.: <http://www.transparency.org/pressreleases_archive/2003/dnld/cpi2003.pressrelease.en. pdf>. Siehe auch: <http://www.es.geocities.com/malversacion>.

Am 10. Februar 2000 hat Jesús Urdaneta Hernández (ehemaliger Putschist von 1992 und langjähriger Chef der politischen Polizei DISIP) der Oberstaatsanwaltschaft zahlreiche substantiierte Korruptionsanschuldigungen gegen Regierungsmitglieder vorgelegt und behauptet, die Regierung sei korrupter als die Altparteien AD und COPEI, siehe: Neuman/McCoy, S. 41. 


\section{Gefährdung des Rechtsstaats}

Nach Ansicht der Opposition verläuft die demokratische Revolution des Präsidenten Chávez nicht mehr im rechtsstaatlichen Rahmen. Die Verfassungsmäßigkeit der Einberufung der ANC wird angezweifelt. Den rechtsstaatlichen Boden verließ die ANC nach Ansicht der Opposition mit ihrem Statut vom August 1999, in dem sie sich zum originären Obersten Staatsorgan erklärte. Damit setzte sich die ANC über die Grenzen des im Wege des Referendums erteilten und zugleich auch begrenzten Mandats hinweg ${ }^{87}$. Hingewiesen wird darauf, dass Gegenstand des Konsultationsreferendums nicht die Schaffung einer originären Obersten Staatsgewalt gewesen sei. Der entsprechende Passus war mit Urteil vom 13. April 1999 vom Obersten Gericht (CSJ) gestrichen worden ${ }^{88}$. Die Opposition wertet die auf der Grundlage der Satzung der ANC erlassenen Dekrete zur Reorganisation der Legislative und Judikative als Usurpation dieser Gewalten sowie der Verfassung von 1961 und als Staatsstreich ${ }^{89}$.

Die Einberufung der ANC wurde auf Art. 181 LOSPP und auf das aus Art. 50 der Verfassung von $1961^{90}$ abgeleitete Recht zur politischen Partizipation gestützt ${ }^{91}$. Mit Urteil vom 18. März 1999 entschied die CSJ, dass ,die Strukturierung der ANC an den Geist der Verfassung von 1961 gebunden sei, welche die Bedingungen für die Einberufung einer solchen Versammlung enthält, weshalb die Einberufung einer ANC nicht die Veränderung der fundamentalen Prinzipien des Rechtsstaats bedeuten kann““92. Die Aufgabe zur Schaffung einer neuen Rechtsordnung konkretisierte das Gericht im genannten Urteil folgendermaßen: „Die ANC soll den Staat durch einen Mechanismus konsolidieren, der die Praxis einer sozialen und partizipativen Demokratie ermöglicht, wobei die neue Verfassung die Erwar-

Brewer-Carías, S. 31f.. Ebenso Sosa Gómez, die zurückgetretene Präsidentin der CSJ: <http:// www.analitica.com/bitblioteca/csj/renuncia.asp>.

Siehe oben Fn. 29. Erst der im Dezember 1999 neu geschaffene TSJ hat die vom Präsidenten und der ANC behauptete Eigenschaft der ANC als originäres Oberstes Staatsorgan bestätigt, vgl. die Urteile der Sala Constitucional Nr. 04 vom 26. Januar 2000 und Nr. 180 vom 28. März 2000 und ging damit über die Entscheidung der CSJ vom 21. Juli 1999 hinaus (oben Fn. 33).

Beispielsweise César Pérez Vivas (COPEI) und Ex-Präsident Carlos Andrés Pérez, <http://www. diariolarepublica.com/agosto/edicion/26/paginas/mundo04.htm >. Der Präsident der Abgeordnetenkammer des Kongresses, Henrique Capriles Radonski, erhob Nichtigkeitsklage und Verfassungsbeschwerde gegen das Dekret vom 25. August der ANC zur Reorganisation der Legislativen, siehe: <http://www.analitica.com/bitblioteca/hcapriles/recurso.asp> und <http://www.bbs.ingedigit.com/constituyente/capriles.htm>.

Art. 50 CN (1961): „Die Aufzählung der in dieser Verfassung enthaltenen Rechte und Garantien darf nicht verstanden werden als Verneinung anderer, die obwohl sie der menschlichen Person zu eigen sind, nicht ausdrücklich aufgeführt sind. Das Fehlen einer gesetzlichen Regelung dieser Rechte mindert nicht ihre Ausübung“. Deutsche Übersetzung, op.cit. (Fn. 13).

92

Brewer-Carías, S. $26 f$.

Urteil Nr. 271 der CSJ/Sala Político-Administrativa vom 18. März 1999, op. cit. (Fn. 19). 
tungen des Volkes befriedigen und zugleich die Vorrausetzungen des demokratischen Verfassungsrechts erfüllen muss, was im wesentlichen die Beibehaltung der fundamentalen Prinzipien des demokratischen Rechtsstaates beinhaltet“. Nach Ansicht von Brewer-Carías ist aus diesem Urteil der CSJ zu folgern, dass die ANC an die Verfassung von 1961 und der darin enthaltenen grundlegenden rechtstaatlichen Prinzipien, wie es beispielsweise die Gewaltenteilung ist, gebunden war ${ }^{93}$. Dies und die Tatsache, dass die ANC nicht dazu ermächtigt war, die neue Verfassung in Kraft zu setzen, lässt nach Ansicht der Opposition den Rückschluss zu, dass die ANC, entsprechend den im Wege des Referendums angenommenen Grundsätzen, nicht dazu ermächtigt war, die unter der Verfassung von 1961 bestehenden Staatsgewalten und Organe abzuschaffen und zu ersetzen.

Bezüglich der neuen Verfassung wird die mangelnde Klarheit über die geltende Fassung und die damit eingetretene Rechtsunsicherheit kritisiert. Seit dem In-Kraft-Treten der Verfassung wurden unter dem Vorwand der Korrektur von Grammatikfehlern, Syntax und Stil von technischen Kommissionen auch inhaltliche Änderungen vorgenommen ${ }^{94}$.

Die Kritik an der Vorgehensweise der ANC setzt sich im Hinblick auf die Etablierung eines Übergangsregimes durch das Dekret vom 22. Dezember 1999 fort $^{95}$. Die Verfassung von 1999 enthielt Übergangsbestimmungen, aus denen sich der Verbleib von Mandatsträgern der öffentlichen Gewalt im Amt bis zur Durchführung von Neuwahlen ergab ${ }^{96}$. Lediglich der Ombudsmann war als neu geschaffenes Organ zu designieren, nicht aber die Richter des TSJ, der Oberstaatsanwalt, der Oberste Rechnungsprüfer und die Mitglieder des Nationalen Wahlrats $(\mathrm{CNE})^{97}$. Die Opposition weist auf die Unterwanderung der Staatsgewalten mit treuen Regierungsanhängern und die damit einhergehende Gefahr der Gleichschaltung hin. Die ANC besetzte auf der Grundlage der Dekrete zur Reorganisation der Legislative und Exekutive und im Rahmen des Übergangsregimes strategische Posten in demokratischen Institutionen mit Gefolgsleuten des Präsidenten. Die Opposition befürchtet eine Vertiefung der Kontrolle der politischen Institutionen durch Regierungsanhänger. Der Vorschlag der Regierungsanhänger, die Richterzahl am TSJ von 20 auf 30 zu erhöhen, wird als Möglichkeit gewertet, die Macht des Präsidenten über das Oberste Gericht zu verfestigen; eine objektive Rechtfertigung für eine solche Erhöhung ist nicht ersichtlich ${ }^{98}$.

93

94

95

96

97

98

Brewer-Carías, S. 27.

Eine Aufzählung der Änderungen findet sich bei: Brewer-Carías, S. 282-289.

Brewer-Carías, S. 32.

Eingehend: Brewer-Carías, S. 253-255.

Siehe hierzu auch: Neuman/McCoy, S. 40.

Vgl. den Protest des Präsidenten der Academia de Ciencias Políticas, Gustavo Planchart und renommierter Juristen gegen diesen Vorschlag, Informe Quincenal de Datanalisis, Marzo 2003: $<$ http://www.datanalisis.com/default.htm>. 


\section{Spaltung der Gesellschaft}

Chávez wird von der Opposition zu Recht vorgeworfen, sozialen Hass geschürt und die venezolanische Gesellschaft in zwei Lager gespalten zu haben. Heute stehen sich die Anhänger der Regierung (das ,gute“ souveräne Volk) und die Anhänger der Opposition (die „,böse“ Oligarchie) unversöhnlich und aufgebracht gegenüber. Die Grenze zwischen den Lagern verläuft durch die Gesellschaft und nicht selten auch durch Familien. Ebenso zerrissen ist das Militär, das in Venezuela keine elitären Züge trägt, sondern klassenübergreifend organisiert ist. Diese Polarisierung gepaart mit Aggression und Hysterie haben dazu geführt, dass sich Venezuela zeitweilig am Rande des Bürgerkrieges bewegte.

\section{Einführung eines autoritären Regimes}

Die Opposition befürchtet, dass die bolivarianische Revolution lediglich ein Vorwand zur Einführung einer Militärdiktatur und der damit einhergehenden Beschränkung verfassungsrechtlicher Freiheiten ist. Nach Ansicht der Opposition bedient Hugo Chávez sich nur dieses Ideengutes, um seine Politik zu rechtfertigen. Ein wahrer linker Revolutionär oder Demokrat ist er nach Auffassung der Opposition nicht, vielmehr verbergen sich hinter dem Populismus des Präsidenten autoritäre Strukturen. Die Opposition weißt darauf hin, dass Chávez sich demokratische und undemokratische Mittel gleichermaßen zu Nutze gemacht hat, um seine Macht abzusichern und zu verstärken. Uslar Pietri prägte den Begriff einer legalen, vom Volk legitimierten Diktatur ${ }^{99}$ und Carrasquero Aumaitre bezeichnete das Regime von Chávez als verfassungsmäßig begründeten und gefestigten populistischen Caudillismus $^{100}$.

In diesem Kontext wird auch die Errichtung von Círculos Bolivarianos, eine Art Nachbarschaftsgruppierungen wie sie in Cuba existieren ${ }^{101}$, gesehen und als Beginn der Einführung einer sozialistischen Autokratie nach kubanischem Vorbild gewertet. Aufgabe der Círculos Bolivarianos ist die Durchdringung der venezolanischen Gesellschaft mit den Ideen und Zielsetzungen der bolivarianischen Revolution ${ }^{102}$. Die Zirkel werden vom Präsidenten organisiert und kommandiert sowie mit öffentlichen Geldern finanziert. Sie sind zum Teil bewaffnet und haben bei mehreren Gelegenheiten ihre Einsatz- und Gewaltbereitschaft bewiesen.

99

100

101 102

Zitiert nach Doménico Chiappe: <http://www.eluniversal.com/verbigracia/memoria/N238/ apertura.shtml>.

Carrasquero Aumaitre, S. 38.

In Cuba sind es die Comités de Defensa de la Revolución (CDR).

Siehe: <http://www.geocities.com/redbolivariana/circulos.htm>. 


\section{Demokratische Lösungen}

Nach Ansicht der Opposition gibt es keine Lösung, ohne Rücktritt des Präsidenten und der Relegitimierung der demokratischen Institutionen. Gemäß der Verfassung von 1999 existieren fünf Optionen, die aufgrund ihrer unterschiedlichen Voraussetzungen, Folgen und abweichenden Verfahrensdauer mit unterschiedlicher Gewichtung von der Opposition eruiert wurden: Das Konsultationsreferendum, das Revokationsreferendum, die Verfassungsänderung, die tiefgreifendere Verfassungsreform und die Einberufung einer Verfassunggebenden Versammlung (ANC). Zudem sind hier die Möglichkeit einer Amtsenthebung und die von Jimmy Carter vorgeschlagenen mediativen Optionen zu nennen.

\section{Konsultationsreferendum, Art. $71 \mathrm{CRBV}$}

Das Oppositionsbündnis setzte zunächst auf ein Konsultationsreferendum über den Rücktritt des Präsidenten Chávez und sich anschließende vorgezogene Neuwahlen. Das Konsultationsreferendum war vom Nationalen Wahlrat (CNE) für den 2. Februar 2003 angesetzt worden $^{103}$. Nachdem jedoch die entsprechende Resolution des CNE vom Obersten Gericht mit Urteil vom 22. Januar 2002 für nichtig erklärt worden war ${ }^{104}$, orientierte sich die Opposition um. Hintergrund für dieses Urteil war die Beteiligung von Leonardo Pizani am Erlass besagter Resolution des CNE. Pizani war im Oktober 2000 von seinem Amt als stellvertretendes Mitglied des CNE zurückgetreten ${ }^{105}$. Am 11. November 2002 hatte Pizani seinen Rücktritt zurückgezogen. Am 18. November 2002 wurde er vom CNE als Mitglied reinkorporiert, um die durch den Rücktritt von Roberto Ruíz (2002) vakante Stelle zu besetzen und die Funktionsfähigkeit des CNE wiederherzustellen ${ }^{106}$. Mit dem Urteil vom 22. Januar „,befahl“ der TSJ dem CNE, davon Abstand zu nehmen, unter Anwesenheit oder Beteiligung von Pizani zu tagen.

Siehe: Resolution Nr. 021203-457 des CNE, veröffentlicht in der Gaceta Electoral Nr. 168 vom 5. Dezember 2002.

Urteil Nr. 3 des TSJ/Sala Electoral Accidental vom 22. Januar 2003.

Siehe: <http://www.ultimasnoticias.com.ve/ediciones/2002/11/15/p16n1.htm> und <http://www. tsj.gov.ve/informacion/notasprensa/notasdeprensa.asp?codigo=257>. Neben Pizani (2000) und Ruíz (2002) waren Imelda Rincón Finol (2000), Cesar Peña Vigas (2000), Ignacio Ávalos (2000) und Vicente Gonzáles Pepper (2002) zurückgetreten. Der Rücktritt von Rincón Finol und Peña Vigas, ebenso wie die darauffolgende Ernennung von José Manuel Zerpa und Roberto Ruíz. wurde veröffentlicht in der Gaceta Oficial Nr. 37.026 vom 31. August 2000.

Nach Art. 29 Estatuto Electoral del Poder Público bedarf es einer qualifizierten Mehrheitsentscheidung von vier der fünf Mitglieder des CNE. Das Statut wurde veröffentlicht in der Gaceta Oficial Nr. 36.884 vom 3. Februar 2000. 


\section{Revokationsreferendum, Art. 72ff. CRBV}

Nach Beendigung des Generalstreiks stand die Durchführung eines Revokationsreferendums im Zentrum der Bemühungen der Coordinadora Democrática. Nach Art. 72 CRBV besteht die Möglichkeit, durch eine Volksabstimmung alle gewählten öffentlichen Amtsträger bis hin zum Staatspräsidenten ihres Amtes zu entheben. Nach Ablauf der Hälfte der Amtsperiode des betreffenden Amtsträgers kann von mindestens 20\% der eingetragenen Wähler (2,4 Mio. bei 12 Mio. eingetragenen Wählern) die Einberufung einer Volksabstimmung über dessen Amtsenthebung beantragt werden. Liegt die Beteiligung am Referendum über 25\% (3 Mio.) und ist dessen Ergebnis, dass eine gleichgroße oder größere Zahl als die Wähler, die den Amtsträger gewählt haben, für dessen Amtsenthebung stimmen, so wird sein Amt als zurückberufen angesehen und das gesetzliche Verfahren zur Neubesetzung des Amtes eingeleitet.

Am 19. August 2003 war die Hälfte des Präsidentschaftsmandats von Chávez erreicht. Chávez wurde im Jahre 2000 bei einer Wahlbeteiligung von 56,5\% mit einer Mehrheit von 59,5\% der Stimmen gewählt. Das zu seiner Absetzung erforderliche Quorum liegt - unter der Voraussetzung einer Wahlbeteiligung von mindestens $25 \%$ - bei $\geq 3.940 .193$ Stimmen $^{107}$. Ist das Ergebnis des Revokationsreferendums positiv und Chávez Mandat widerrufen, tritt der Fall der endgültigen Verhinderung (falta absoluta) des gewählten Präsidenten ein, so dass innerhalb der darauffolgenden dreißig Tage neue, allgemeine und direkte Wahlen abzuhalten sind, Art. 233 CRBV. Bis der neue Präsident gewählt und in sein Amt eingeführt ist, übernimmt der Vize-Präsident die Präsidentschaft. Chávez könnte im Falle der Revokation seines Mandats nach einem Urteil des TSJ vom 28. August 2003 nicht erneut kandidieren $^{108}$.

Nach Angaben einer Studie von Datanalisis würden über $60 \%$ der eingetragenen Wähler Präsident Chávez im Rahmen eines Referendums des Amtes entheben ${ }^{109}$.

Es wurden Vorwürfe erhoben, wonach die Regierung im großen Stil das Verfahren zur Einbürgerung erleichtert (cedulación) und sich davon eine Stimmenabgabe für Chávez im

Die Zahl der eingetragenen Wählerschaft lag bei 11.720 .660 , vgl. den Bericht der OAS: OEA/Ser.D/XX/SG/UPD/II.30, S. 36, <http://www.upd.oas.org/lab/Documents/publications/ electoral_observation/2000/pbl_30_2000_spa.pdf.>. Wenngleich Art. 72 CRBV bestimmt, dass die Zahl der Wähler gleichgroß oder größer sein muss, ist nicht auszuschließen, dass der TSJ den Verfassungstext dahingehend auslegt, dass statt der absoluten Zahl der Prozentsatz von 59,5\% der abgegebenen Stimmen erreicht werden muss.

109 Siehe: <http://www.eluniversal.com/2003/07/29/29072003_72426.html> und die Encuesta Nacional Ómnibus von Datanalisis vom Juli 2003 <http://www.datastrategia.com/elecciones/ atlas/DatanalisisOmnibusMayo2003.pdf>. 
Referendum versprochen haben soll ${ }^{110}$. Ebenso soll die Oficina Nacional de Identificación y Extranjería (Onidex) gefälschte Personalausweise für Chávez Anhänger ausgestellt haben, um ihnen auf diese Weise eine mehrmalige Stimmenabgabe zu ermöglichen ${ }^{111}$.

Mögliche Hindernisse oder zeitliche Verzögerungen für die Durchführung des Revokationsreferendums können sich im Zusammenhang mit der Feststellung des Zeitpunktes der Hälfte der sechsjährigen Amtszeit ergeben. Hugo Chávez trat nach seiner Wahl im Dezember 1998 am 2. Februar 1999 sein Amt an, Art. 186 CN (1961). Am 30. Juni 2000 wurde Chávez wieder gewählt und legte am 19. August 2000 erneut den Amtseid ab. Nach Art. 231 CRBV (1999) tritt ein gewählter Präsident am 10. Januar des ersten Jahres seiner Amtsperiode sein Amt an. Der Oberste Gerichtshof könnte deshalb feststellen, dass nicht der 19. August 2003, sondern erst der 10. Januar 2004 (Amtszeit: 10. Januar 2001 - 10. Januar 2007) die „Halbzeit“ markiert ${ }^{112}$. Ist die Hälfte der Amtszeit erreicht, kann die Durchführung des Referendums beantragt werden. Die Opposition hat am 2. Februar 2003 nach eigenen Angaben rund 3,24 Mio. Unterschriften eingesammelt (el firmazo) ${ }^{113}$ und am 20. August 2003 in ausreichender Menge beim CNE eingereicht. Der CNE entschied, dass die Unterschriftensammlung im Februar zur unangebrachten Zeit erfolgt sei, da sie erst ab Hälfte der Amtszeit rechtmäßig sei ${ }^{114}$. Begründet wurde diese Entscheidung mit dem Argument, dass das Recht zur Durchführung des Referendums zum Zeitpunkt der Unterschriftensammlung noch nicht entstanden gewesen sei. Dem ließe sich jedoch entgegenhalten, dass, wenn das Einreichen der Unterschriften ab der Hälfte der Amtszeit möglich ist, eine vorherige Unterschriftensammlung zulässig sein muss. Die Opposition reagierte mit der

Siehe: El Universal vom 11. Mai 2003. Vgl. auch: <http://www.militaresdemocraticos.com/ articulos/sp/20030201-04.html> und <http://www.militaresdemocraticos.com/denuncias/sp/ 20021112-19.html>.

Siehe das Dossier des Journalisten Bocaranda Sardi: <http://www.militaresdemocraticos.com/ articulos/sp/20030201-04.html>. Besondere Aufmerksamkeit fand der gefälschte Personalausweis (cédula de identidad) von Rodríguez Chacín, dem Ex-Minister für Justiz und Inneres, vgl.: <http://www.eluniversal.com/2002/05/29/29052002_31140.html>.

Zwar hat der TSJ/Sala Constitucional wiederholt festgestellt, dass die Hälfte der Amtszeit am 19. August 2003 beginnt (beispielsweise im Urteil Nr. 137 vom 13. Februar 2003), dennoch beinhaltete der Vorschlag von Carter in weiser Voraussicht, die Bestätigung dieses Datums durch das Gericht. Zu den Vorschlägen von Carter siehe unten Fn. 129.

Siehe: <http://www.sumate.com/centros.asp?c=noticias>. Die Organisation Súmate koordinierte den firmazo. Den venezolanischen Bürgern wurden insgesamt zehn Formulare (plantillas) in Wahllokalen zur Unterschrift vorgelegt. Das Formular Nr. 2 betraf die Verkürzung der Präsidentschaftsmandats auf 4 Jahre, das Formular Nr. 7 das Revokationsreferendum, das Formular Nr. 9 die Einberufung einer Verfassunggebenden Nationalversammlung, vgl.: <http:// noticiero.venevision.net/firmazo/plantilla.htm>.

Die Resolution Nr. 030912-461 des CNE vom 12. September 2003 ist abgedruckt in der Gaceta Oficial Nr. 37.776 vom 16. September 2003. Die eingereichten Unterschriften wurden vom CNE als extemporáneas bezeichnet. 
Planung einer weiteren Unterschriftensammlung (el reafirmazo). Dem erneuten Einreichen von Unterschriften könnte jedoch Art. 72 CRBV entgegenstehen. Hiernach kann während der Periode für die ein Amtsträger gewählt worden ist, nicht mehr als ein Antrag auf Revokation seines Mandats gestellt werden ${ }^{115}$. Der Normzweck von Art. 72 CRBV liegt darin, dem Missbrauch dieses neu eingeführten Rechts vorzubeugen. Der Normzweck kann jedoch nicht darin liegen, die Ausübung dieses Rechts dadurch zu erschweren, dass formal unzulässige Anträge als Anträge im Sinne der Norm aufzufassen sind, mit der Folge, dass in der gesamten Amtsperiode eine erneute Antragstellung ausgeschlossen ist. Für die Zulässigkeit eines weiteren Antrages während der laufenden Amtsperiode von Präsident Chávez spricht zudem, dass die Durchführung des Referendums integraler Bestandteil des im Mai 2003 abgeschlossenen Abkommens zwischen Regierung und Opposition ist ${ }^{116}$.

\section{Verfassungsänderung, Art. 340ff. CRBV}

Eine Möglichkeit, die Verkürzung des Präsidentschaftsmandats von 6 auf 4 Jahre und anschließende Neuwahlen herbeizuführen, liegt in der Verfassungsänderung. Im Rahmen dieses Verfahrens werden einzelne oder mehrere Artikel verändert oder hinzugefügt, ohne jedoch die grundlegende Struktur der Verfassung anzutasten.

Das Initiativrecht steht neben dem Präsidenten im Ministerrat und $30 \%$ der Abgeordneten auch $15 \%$ (ca. 1,8 Mio.) der im Wahlregister eingetragenen Bürger zu. Dreißig Tage nach Eingang des Reformentwurfs beruft die Wählergewalt (ausgeübt durch den CNE), ein Annahmereferendum (referendo aprobatorio de leyes) ein. Stimmen $25 \%$ (ca. 3 Mio.) der eingetragenen Wähler für die Verfassungsänderung, gilt sie als angenommen, Art. 341 Abs. 4 i.V.m. Art. 73 CRBV. Die Verfassungsänderung ist vom Präsidenten binnen der darauffolgenden zehn Tage als Gesetz zu erlassen, Art. 346 CRBV. Geschieht dies nicht, so werden die Änderungen vom Präsident und den beiden Vize-Präsidenten des Parlaments erlassen, Art. 216 CRBV. Im Rahmen des firmazo wurden am 2. Februar 2003 rund 4,5 Mio. Unterschriften für die Verfassungsänderung zur Verkürzung des Präsidentschaftsmandats eingesammelt ${ }^{117}$. Es stellt sich jedoch die Frage, ob die Verkürzung des Präsidentschaftsmandats überhaupt im Wege der bloßen Verfassungsänderung möglich ist, da wesentliche Änderungen nur im Wege der Verfassungsreform zu erreichen sind. Wird diese Frage dem Obersten Gericht zur Entscheidung vorgelegt, ist es denkbar, dass es den Änderungsvorschlag als Verfassungsreform deklariert. Stellt es hingegen fest, dass es sich um eine Verfassungsänderung handelt, könnte eine Verkürzung der gegenwärtigen Amtzeit von

Art. 72 CRBV: "Durante el período de para el cual fue elegido el funcionario o funcionaria no 116 podrá hacerse más que una solicitud de revocación de su mandato".

117 Siehe unten C VII.

Siehe oben Fn. 112. 
Chávez daran scheitern, dass der Änderung keine Rückwirkung beigemessen wird und sie erst die folgende Amtsperiode gilt.

\section{Verfassungsreform, Art. 342ff. CRBV}

Durch eine Verfassungsreform können ebenfalls die Verkürzung des Präsidentschaftsmandats und sich anschließende Neuwahlen herbeigeführt werden. Diese Option ist aufgrund ihrer langen Dauer von der Opposition nicht in Betracht gezogen worden. Der Reformentwurf muss drei Lesungen im Parlament unterzogen werden. Die Nationalversammlung hat, gerechnet ab Zugang und Annahme des Reformgesuchs, zwei Jahre Zeit, um zu entscheiden. Ferner muss der Entwurf mit einer Zwei-Drittel-Mehrheit im Parlament angenommen werden, um Gegenstand eines dreißig Tage später einzuberufenden Annahmereferendums (referendo aprobatorio) zu werden, Art. 343f. CRBV.

\section{Verfassunggebende Nationalversammlung, Art. 347ff. CRBV}

Die Asamblea Nacional Constituyente (ANC), die mit dem Ziel der Umwandlung des Staates, der Schaffung einer neuen Rechtsordnung und der Redaktion einer neuen Verfassung einberufen werden kann, stellt ebenfalls ein Instrument zur Herbeiführung einer Ver kürzung der Amtsperiode des Präsidenten und sich anschließender Neuwahlen dar. Das Initiativrecht steht neben dem Präsidenten im Ministerrat und $30 \%$ der Abgeordneten, $15 \%$ (ca. 1,8 Mio.) der im Wahlregister eingetragenen Bürger zu. Die Verfahrensgrundsätze zur Wahl der Mitglieder der ANC sind Bestandteil der Stimmenabgabe bei Ausübung des Initiativrechts. Nach Art. 349 CRBV ist es dem Präsidenten nicht möglich, Einspruch gegen die neue Verfassung zu erheben. Den Staatsgewalten ist es nicht möglich, auf die Entscheidungen der ANC Einfluss zu nehmen. Auch diesen Weg hat die Opposition zunächst eingeschlagen und die hierfür erforderlichen Unterschriften im Rahmen des firmazo am 2. Februar 2003 eingesammelt ${ }^{118}$. Hierbei ist die Opposition im Hinblick auf die Formulierung der bases comiciales dem Vorbild der Grundsätze gefolgt, nach denen die Verfassungsgebende Versammlung im Jahre 1999 einberufen wurde, um sich nicht dem Vorwurf der Verfassungswidrigkeit auszusetzen. Diese Unterschriften wurden bisher nicht eingereicht. 


\section{Amtsenthebung, Art. 266 Nr. 2 i.V.m. Art. 233 CRBV}

Parallel zu den geschilderten Bemühungen wird auch ein Verfahren zur Amtsenthebung von Hugo Chávez betrieben ${ }^{119}$. Die gegen ihn beim Obersten Gericht (TSJ) eingereichten Strafanzeigen ${ }^{120}$ reichen von der Veruntreuung und Unterschlagung öffentlicher Mittel des Investitionsfonds für die makroökonomische Stabilisierung (FIEM) ${ }^{121}$, über die Annahme illegaler Spenden für seinen Wahlkampf 1998/1999 vom Banco Bilbao Vizcaya Argentaria $(\mathrm{BBVA})^{122}$ und vom Banco de Santander ${ }^{123}$, bis hin zur rechtswidrigen Finanzierung der bolivarianischen Zirkel durch öffentliche Mittel des Fondo Intergubernamental para la Descentralización (FIDES) ${ }^{124}$. Hinzu kommen schwere Vorwürfe wegen Verbrechen gegen die Menschlichkeit (lesa humanidad) ${ }^{125}$ und der Verletzung von Menschenrechten im Zusammenhang mit den Geschehnissen des 11. April 2002 ${ }^{126}$. Ferner der Vorwurf der Schädigung des Staatsvermögens durch ein Kooperationsabkommen mit Cuba, wonach an Cuba täglich 53.000 Fässer Erdöl zu Vorzugspreisen im Austausch gegen die Bereitstellung von Dienstleistungen und Personal (Ärzte, Lehrer, Techniker, Sporttrainer etc.) geliefert werden ${ }^{127}$. Die Erfolgsaussichten eines Amtsenthebungsverfahrens sind gering, denn selbst wenn der TSJ das Vorliegen hinreichender Tatsachen (antejuicio de méritos) für eine Anklageerhebung feststellen würde, unterliegt der Fortgang des Verfahrens einer vorherigen Autorisierung seitens des von Regierungsanhängern dominierten Parlaments.

Siehe oben Fn. 6.

${ }^{120}$ Eine Chronologie findet sich unter: <http://politica.eluniversal.com/informespecial/juicioalpresidente/cronologia.shtml>. Siehe auch: <http://www.venezuelalibre.net/analisis199.20021127. html>.

121

Die Strafanzeige von Andrés Velásquez, Elías Matta Wehbe, Enrique Márquez und Alejandro Armas (Causa R) wurde am 27. Juni 2002 beim TSJ/Sala Penal eingereicht, siehe oben Fn. 120.

Die von Tulio Alberto Álvarez am 20. Juni 2002 gestellte Strafanzeige wurde vom TSJ/Sala Penal mit Urteil Nr. 39 vom 24. September 2002 mangels hinreichender Beweise nicht weiter verfolgt. Tulio Álvarez hat Berufung eingelegt. Es folgten weitere Strafanzeigen von Enrique Ochoa Antich (14. Juni 2002) und Oscar Bohórquez (27. Juni 2002), siehe oben Fn. 120.

123 Von Henry Ramos Allup und Rafael Marín (AD) am 10. Juli 2002 eingereichte Anzeige, siehe oben Fn. 120.

124 Von Enrique Ochoa Antich, Raúl Pinto Peña, und weiteren anderen am 10. Juni 2002 eingereichte Anzeige, siehe oben Fn. 120.

125 Die Anzeige der Familienangehörigen der Opfer und der Asociación Civil Fuerza Integradora vom 25. Juni 2002 wurde auf Eis gelegt, bis eine Entscheidung der Kammer für Verfassungssachen über das Tätigwerden der Generalstaatsanwaltschaft in diesem Zusammenhang vorliegt, siehe oben Fn. 120.

126 Von Felipe Mujica, Leopoldo Puchi und Carlos Tablante (MÁS) am 2. Juli 2002 eingereichter Antrag, siehe oben Fn. 120.

127

Die von Felipe Mujica und Leopoldo Puchi eingereichte Nichtigkeitsklage wurde vom TSJ/Sala Constitucional mit Urteil Nr. 2911 vom 20. November 2002 als unbegründet abgewiesen. Alejandro Terán hat am 26. Juni 2002 ebenfalls Klage eingereicht, siehe oben Fn. 120. 


\section{Vorschläge von Jimmy Carter}

Die Staatskrise in Venezuela ist Gegenstand internationaler Mediation. Am Verhandlungstisch, der Mesa de Negociaciones y Acuerdos, zwischen Regierung und Opposition agierte neben dem früheren US-Präsidenten und Friedensnobelpreisträger Jimmy Carter auch OAS-Generalsekretär César Gaviria ${ }^{128}$. Carter unterbreitete im Januar 2003 in Caracas zwei Vorschläge zur politischen Beilegung der Krise: Die verhandelte Verfassungsänderung und das verhandelte Revokationsreferendum ${ }^{129}$. Beide Vorschläge zeichneten sich dadurch aus, dass sie zum Teil auf der Grundlage einer vorhergehenden Einigung zwischen Regierung und Opposition von den verfassungsrechtlichen Vorgaben abwichen. Voraussetzung für beide Vorschläge war, dass die Opposition den Generalstreik beendet und die Regierung versichert, dass es keinerlei Repressalien gegen die wegen ihrer Beteiligung am Streik zu Tausenden entlassenen Angestellten der PDVSA geben werde. Die Opposition beendete im Februar 2003 den Generalstreik, der einen wirtschaftlichen Schaden in Milliardenhöhe an US\$ verursachte. Die Regierung ist der an sie gerichteten Verpflichtung bislang nicht nachgekommen. Vielmehr ergingen richterliche Haftbefehle gegen die Anführer des Generalstreiks Carlos Ortega (Vorsitzender des CTV) und Carlos Fernández (Präsident der Fedecámaras) ${ }^{130}$.

Erreicht wurde die Redaktion eines Vor-Abkommens zwischen Regierung und Opposition, das der Öffentlichkeit am 11. April 2003 präsentiert wurde ${ }^{131}$. Am 29. Mai 2003 unterzeichneten Regierung und Opposition ein Abkommen, das neben dem Revokationsreferendum des Präsidenten weitere Revokationsreferenda gegen alle gewählten Amtsträger vorsieht $^{132}$. Die Durchführung des Referendums, konnte im Abkommen nicht wie beabsichtigt für August 2003 festgelegt werden. Vielmehr wurde die Terminierung der Abstimmungen dem damals noch zu wählenden CNE aufgetragen ${ }^{133}$.

Unterstützt werden die internationalen Bemühung des Weiteren vom Centro Carter (CC), vom Programa de Naciones Unidas para el Desarrollo (PNUD) und von der am 24. Januar 2003 vom brasilianischen Staatschef Luiz Inácio Lula da Silva ins Leben gerufenen Gruppe der "Freunde 129 Venezuelas" (Brasilien, Chile, Mexiko, Portugal, Spanien und die USA).

Die Vorschläge von Carter finden sich unter: <http:// www.oas.org/OASpage/eng/Ve_infCarter 012103-esp.htm>.

130 Vorgeworfen wird ihnen unter anderem Widerstand gegen die Staatsgewalt, Landesverrat und Anstiftung zur Begehung einer Straftat, vgl. <http://www.eluniversal.com/2003/02/20/20022003 _45948.html>. Die Haftbefehle werden als Verstoß gegen den kurz zuvor am 18. Februar 2003 zwischen Regierung und Opposition vereinbarten „Pakt gegen Gewalt, für den Frieden und die Demokratie" gewertet.

131 Siehe: <http://www.oas.org/OASpage/eng/Venezuela/acurdo-041103.htm>.

132 Siehe Nr. 12 des Abkommens: <http://www.oas.org/defaultesp.htm>.

133

Siehe Nr. 17 des Abkommens: <http://www.oas.org/defaultesp.htm>. Zur Ernennung der Mitglieder des CNE siehe oben Fn. 64. 


\section{Ausblick}

Das Revokationsreferendum ist eine Feuerprobe der bolivarianischen Revolution und der demokratischen Kräfte in Venezuela. Hugo Chávez erklärte sich im April 2003 mit dem Referendum einverstanden: Er habe keine Angst davor, als erster venezolanischer Präsident sein Volk über seine politische Zukunft entscheiden zu lassen. Eine tatsächliche Bereitschaft der Regierung zur Ermöglichung des Referendums ist indes nicht zu erkennen. Vielmehr wirkt die Diskussionsbereitschaft der Regierung Chávez ebenso plakativ wie deren Wille zur Beilegung der Krise. Dies mag daran liegen, dass das für Präsident Chávez günstigste Zukunftsszenario in der Verzögerung des Referendums liegt. Fällt das Referendum in seine beiden letzten Amtsjahre, so übernimmt im Falle seiner Absetzung bis zur Vollendung des sechsjährigen Präsidentschaftsmandats sein Vize-Präsident das Amt, Art. 233 CRBV. In diesem Fall wird sich nichts am Kurs der Regierung Chávez ändern, denn Vize-Präsident José Vicente Rangel wurde vom Präsidenten persönlich ernannt. Der Einfluss von Chávez auf die entscheiden den Institutionen ist ausreichend, um das Referendum in diesem Sinne zu verzögern.

Findet das Referendum vor dem 19. August 2004 statt, ist eine Lösung des venezolanischen Konflikts jedoch nicht gewährleistet, denn die Krise geht weiter als ihre Ursachen. Die Opposition ist lediglich in der Ablehnung des amtierenden Präsidenten geeint, verfügt jedoch weder über einen Kandidaten ${ }^{134}$, der das Vertrauen der Venezolaner gewinnen könnte, noch über ein einheitliches Programm. Diese Uneinigkeit der Opposition spielt Chávez freilich in die Hände. Trotz starker Popularitätseinbußen wird er nach wie vor von den unteren Sozialschichten gestützt und ist deren Hoffnungsträger. Im Falle der Durchführung des Referendums und einer Abwahl des Präsidenten bleibt zu hoffen, dass das Land nicht in eine noch tiefere Krise stürzt, sondern dessen Probleme grundlegend angepackt werden. des Staates Carabobo) können zwar viele Stimmen auf sich vereinen, ob dies im Ernstfall ausreichen würde, ist ungewiss. 